\title{
Antibiotic Resistance Pattern of Acinetobacter baumannii Strains Isolated from Different Clinical Specimens and Their Sensibility Against Bioactive Molecules Produced by Actinobacteria
}

\author{
Victoria Namiganda ${ }^{1,2} \cdot$ Yousra Mina $^{1,2} \cdot$ Atika Meklat $^{1,2} \cdot$ Djamila Touati $^{3} \cdot$ Noureddine Bouras $^{2,4}(\mathbb{D})$ \\ Mustapha Barakate ${ }^{5}$. Nasserdine Sabaou ${ }^{2}$
}

Received: 29 December 2018 / Accepted: 16 April 2019 / Published online: 9 May 2019

(c) King Fahd University of Petroleum \& Minerals 2019

\begin{abstract}
Acinetobacter baumannii frequently targets the most vulnerable hospitalized patients, especially in intensive care units. Bacteremia, nosocomial pneumonia and surgical wound infections caused by A. baumannii have a higher mortality compared to other nosocomial pathogens. The severity of the diseases caused by this microorganism is accentuated by its resistance to many antibiotics currently used in the world. In this context, we undertook to isolate several A. baumannii strains from various samples collected in the Béni Messous University Hospital Center, Algiers (Algeria), to determine the antibiotic resistance profile of these strains and to evaluate the antagonistic potential of some actinobacterial strains against A. baumannii. Nineteen A. baumannii strains were isolated and identified. The predominant number of strains isolated from pulmonary samples (36.84\%) and from the intensive care unit (57.89\%) was A. baumannii. All A. baumannii strains were sensitive to colistin but exhibited high resistance to 11 other antibiotics tested. Of the 19 strains, 15 were metallo- $\beta$-carbapenemase producers, 10 were cephalosporinase, and three were extended-spectrum $\beta$-lactamases (ESBL) producers. Of 59 strains of actinobacteria tested, 19 (32.20\%) showed activity against at least one strain of A. baumannii, the highest activity being detected in strains belonging to the genus Saccharothrix (or related genera). However, Streptomyces sp. WAB9 had the widest spectrum of activity. Based on the obtained results, colistin remains the unique antibiotic which inhibits the growth of all A. baumannii strains, and some actinobacterial strains also present an alternative source of new molecules with strong bioactivities against multidrug-resistant A. baumannii strains.
\end{abstract}

Keywords Acinetobacter baumannii · Antibiotic $\cdot$ Antibiotic resistance $\cdot$ Actinobacteria $\cdot$ Antagonism

Noureddine Bouras

noureddine_bouras@yahoo.fr

1 Département de Biologie et Physiologie Cellulaire, Faculté des Sciences de la Nature et de la Vie, Université Saâd Dahleb, Blida 1, Algeria

2 Laboratoire de Biologie des Systèmes Microbiens, Ecole Normale Supérieure de Kouba, Algiers, Algeria

3 Laboratoire de Microbiologie du Centre Hospitalo-Universitaire de Béni Messous, Algiers, Algeria

4 Département de Biologie, Faculté des Sciences de la Nature et de la Vie et Sciences de la Terre, Université de Ghardaïa, BP 455, Ghardaïa 47000, Algeria

5 Laboratoire Biologie et Biotechnologie des Microorganismes (LBBM), Faculté des Sciences-Semlalia, Université Cadi Ayyad, BP 2390, Marrakech, Morocco

\section{Introduction}

Bacterial species belonging to the genus Acinetobacter, particularly Acinetobacter baumannii, have received increasing attention in recent years because of their potential to cause serious infections and for their ability to rapidly develop resistance to antibiotics. In fact, in the space of 40 years, this species have gone from being an "uninteresting bacterium" because it was not very pathogenic and happened to be sensitive to most of the antibiotics available at that time, to a pathogenic bacterium easily capable of acquiring multiresistance to antibiotics [1].

The majority of infections caused by A. baumannii are contracted in hospitals, most often in critically ill patients hospitalized in intensive care or surgery [2]. In the 1970s, hospital-acquired pneumonia was the most common infection caused by this bacterium. Peleg et al. [3] reported that 
infections involving the central nervous system, skin, bone and soft tissues have appeared to be very problematic for some institutions.

The ability of this opportunistic pathogen to survive in the environment under adverse conditions for prolonged periods may have contributed to its endemic and epidemic behavior. This is also attributed to a few important factors which include the resistance of this agent to disinfectants, desiccation, nutritional deprivation in the moist environment and biofilm formation [4]. In addition, the persistent presence of A. baumannii in hospitals causes it to come into contact with antibiotics which exert a selective pressure favoring strains with antibiotic resistance [5].

In view of antibacterial resistance, the current work is directed toward the search for new antibiotics. Actinobacteria phylum occupies a prominent place among the microorganisms of interest in various fields, particularly in the medical field $[6,7]$. They are able to secrete many bioactive molecules, the most important of which are antibiotics [8]. Our study aims to characterize the antibiotic resistance mechanisms in A. baumannii strains isolated at the Béni-Messous hospital (Algiers, Algeria) and to analyze the antagonistic activity of actinobacterial isolates against these pathogenic strains.

\section{Materials and Methods}

\subsection{Sample Collection}

Over the course of this study (1st February-30th May, 2018), a total of 664 samples were collected from patients in different services (reanimation, pediatrics and nephrology) and the outpatient department at the Béni Messous University Hospital Center. Specimens analyzed included blood (122), catheter (34), pus (198) and pulmonary (310).

\subsection{Isolation and Identification of Acinetobacter baumannii Strains}

All collected samples were cultured on blood and chocolate agar by the 4-quadrant streak plate method [9]. After $18 \mathrm{~h}$ of incubation (at $35^{\circ} \mathrm{C}$ ), pure cultures of presumptive A. baumannii were first subjected to the standard bacteriological techniques (morphology, Gram stain, catalase test and oxidase test, and MEVAG medium used to determine the pathway of attack of glucose and to test the carbohydrates used by oxidative bacteria) and identified later as A. baumannii by commercial API 20NE multitest system (BioMérieux, Vitek, Marcy l'Etoile France) [10-12]. This biotyping method is a standardized system for the identification of non-fastidious, non-enteric and non-fermentative Gram-negative rods, combining eight conventional tests which include potassium nitrate $\left(\mathrm{NO}_{3}\right)$, L-tryptophane (TRP), D-glucose (GLU), L-arginine (ADH), urea (URE), esculin ferric citrate (ESC), gelatin (GEL) and 4-nitrophenyl- $\beta$-D-galactopyranoside (PNPG) and also assimilation of 12 compounds, which include D-glucose (GLU), L-arabinose (ARA), D-mannose (MNE), D-mannitol (MAN), N-acetyl-glucosamine (NAG), D-maltose (MAL), potassium gluconate (GNT), capric acid (CAP), adipic acid (ADI), malic acid (MLT), trisodium citrate (CIT) and phenylacetic acid (PAC). Wells of biochemical test were inoculated with $0.5 \mathrm{McF}$ arland bacterial suspension and incubated for $48 \mathrm{~h}$ (at $37^{\circ} \mathrm{C}$ ). The results were read after addition of appropriate reagents, as 7-digit number that identify by API 20NE analytical index.

\subsection{Antibacterial Resistance Study}

Antibacterial resistance of A. baumannii strains was determined for 11 antibiotics by the disk diffusion method. Sensitivity or resistance of each strain to the antibacterial drugs was determined according to the guidelines of the Clinical and Laboratory Standard Institute (CLSI) [13]. Antibiotics tested were ticarcillin (75 $\mu \mathrm{g}$ ), piperacillin $(100 \mu \mathrm{g})$, ticarcillin + clavulanic acid $(75 / 10 \mu \mathrm{g})$, ceftazidime $(30 \mu \mathrm{g})$, imipenem $(10 \mu \mathrm{g})$, ciprofloxacin (5 $\mu \mathrm{g}$ ), trimethoprim/sulfamethoxazole (cotrimoxazole: $1.25 / 23.75 \mu \mathrm{g})$, gentamicin $(10 \mu \mathrm{g})$, amikacin $(30 \mu \mathrm{g})$, tobramycin $(10 \mu \mathrm{g})$ and doxycycline $(30 \mu \mathrm{g})$.

Minimum inhibitory concentration (MIC) of colistin (polymyxin E) was determined by agar dilution method according to the recommendations of CLSI. Briefly, MuellerHinton (MH) agar containing different concentrations of colistin which ranged from 0.125 to $256 \mu \mathrm{g} / \mathrm{ml}$ was inoculated with a bacterial suspension whose turbidity was adjusted to 0.5 McFarland.

\subsection{Antagonistic Activity of Actinobacteria Against A. baumannii}

A total of 59 actinobacterial isolates were tested for antagonistic activity against the 19 A. baumannii strains using the agar plug diffusion method. The actinobacterial isolates were provided by the Laboratory of Biology of Microbial Systems of École Normale Supérieure of Kouba (Algiers, Algeria). They were isolated from Algerian soils and sediments. These Actinobacteria belonged to Saccharothrix and other closely related genera (13 isolates), Actinomadura (27 isolates) and Streptomyces (19 isolates). An agar culture of the Actinobacteria was made on ISP2 medium (yeast extract: $4 \mathrm{~g}$, malt extract: $10 \mathrm{~g}$, glucose: $4 \mathrm{~g}$, distilled water: $1 \mathrm{~L}, \mathrm{pH}$ : 7.2) by tight streaks on the plate surface and incubated for 10 days (at $\left.30^{\circ} \mathrm{C}\right)$. The agar cylinders $(10 \mathrm{~mm}$ in diameter) were cut aseptically with a sterile cork borer from these Acti- 
nobacteria cultures and deposited on the agar surface of $\mathrm{MH}$ plates previously inoculated by A. baumannii ( $0.5 \mathrm{McFar}-$ land), then incubated for $18 \mathrm{~h}\left(\right.$ at $\left.35^{\circ} \mathrm{C}\right)$. After incubation, the antibacterial activity was evaluated by the measuring of the inhibition zone around the agar [14].

\subsection{Testing for the ESBL Production}

ESBL's production was tested by the Double Disk Synergy Test (DDST), using a disk of ticarcillin + clavulanic acid along with ceftazidime (cephalosporin). The bacterial strains were cultured on $\mathrm{MH}$ agar plates, as recommended by CLSI. A disk which contained ticarcillin + clavulanic acid $(75 / 10 \mu \mathrm{g})$ was placed on the plate at a distance of $25 \mathrm{~mm}$ from that of ceftazidime $(30 \mu \mathrm{g})$ and allowed to diffuse at room temperature (for $1 \mathrm{~h}$ ). Once the time had elapsed, the disk of ticarcillin + clavulanic acid was replaced by another disk of ceftazidime. The plate was incubated for 18-24 h (at $35^{\circ} \mathrm{C}$ ). An increase greater or equal to $5 \mathrm{~mm}$ in the inhibition diameter of the ceftazidime disk applied after pre-diffusion of ticarcillin + clavulanic acid in comparison with the ceftazidime disk alone, was considered as positive for ESBL production [13].

\subsection{Cephalosporinase Detection}

All the bacterial strains were tested for cephalosporinase production by cloxacillin test. A suspension of $0.5 \mathrm{McF}$ arland was prepared and inoculated on a MH agar plate, supplemented with cloxacillin $(500 \mathrm{mg})$. The disks of ceftazidime $(30 \mu \mathrm{g})$, tobramycin $(10 \mu \mathrm{g})$, piperacillin $(100 \mu \mathrm{g})$, ticarcillin $(75 \mu \mathrm{g})$, cefepime $(30 \mu \mathrm{g})$ and imipenem $(10 \mu \mathrm{g})$ were placed on the inoculated surface of the agar. After $18 \mathrm{~h}$ of incubation $\left(\right.$ at $35^{\circ} \mathrm{C}$ ), the plates were examined for an increase in the diameter of the zone of inhibition around the disk of ceftazidime which indicated enzyme inactivation (positive result), as compared to the ceftazidime disk on the MH agar plate without cloxacillin [13].

\subsection{Testing for Metallo- $\beta$-carbapenemase Production}

EDTA test was used for the phenotypic detection of metallo$\beta$-carbapenemase. Two $10 \mu \mathrm{g}$ imipenem disks were placed on $\mathrm{MH}$ agar, and $4 \mu \mathrm{l}$ of EDTA (ethylenediaminetetraacetic acid) solution was added to one of the disks. The inhibition zones of the imipenem disk and imipenem + EDTA disk were compared after 18 to $24 \mathrm{~h}$ of incubation (at $35^{\circ} \mathrm{C}$ ). An increased inhibition zone of the imipenem-EDTA disk compared to the imipenem disk alone was considered as MBL positive [13].

All the bacterial strains were also subjected to Hodge test for detection of metallo- $\beta$-carbapenemases. A 0.5 McFar- land of $E$. coli ATCC 25922 suspension was prepared in $5 \mathrm{ml}$ of sterile saline solution $(0.9 \% \mathrm{NaCl}$, at room temperature $)$, then $0.5 \mathrm{~mm}$ of the Escherichia coli ATCC 25922 suspension was added to $4.5 \mathrm{ml}$ of saline solution to make a $1: 10$ dilution. Diluted suspension was inoculated on a MH plate by streaking over the entire plate with the help of a sterile cotton swab. A $10 \mu \mathrm{g}$ imipenem disk was placed in the center of the MH plate. Radial streaks of the test organism, positive control (A. baumannii carbapenemase positive) and negative control (E. coli ATCC 25922), were done from the edge of the imipenem disk to the edge of the plate and then incubated (at $35^{\circ} \mathrm{C}$ ) in an ambient air for 18-24 h. Metallo- $\beta$ carbapenemases production was detected by the appearance of the enhanced $E$. coli ATCC 25922 growth along the test organism that revealed a clover-leaf-like indentation which indicated a positive test $[10,13]$.

\section{Results and Discussion}

\subsection{Characteristics of A. baumannii Strains}

Macroscopic examination of cultures showed smooth, circular, convex and whitish colonies. Microscopic examination after Gram differential staining revealed small bacilli or coccobacilli bacteria, with mixed (variable) staining (mainly pink and purple), rounded ends, isolated or grouped in two or in short chains. Enzymatic tests performed on the bacterial colonies presumptively identified as Acinetobacter (basing on morphological characterization and Gram stain), revealed a negative reaction to oxidase (oxidase -), positive reaction to catalase (catalase + ) and oxidative degradation of sugars on MEVAG medium. All bacterial strains with the above characteristics were identified by API $20 \mathrm{NE}$ (based on biochemical tests), which is widely used in hospital laboratories. After reading and interpretation of the API 20 NE strip, only 19 strains revealed very good identification level of A. baumannii with 7-digit number (0041072) according to the analytical profile index. Seven analytic profile index numbers that we have found $(0041453,000473,0041173,0041072,0043073$, 0045073, 0040053) have been reported for clinical and environmental A. baumannii strains [15]. Henein [16] reported two other analytic profiles: 0001073 and 1041073, which we also have detected.

\subsection{Distribution of A. baumannii Strains According to the Nature of Samples}

A total of 19 strains of A. baumannii were isolated from a variety of clinical samples as shown in Fig. 1. The obtained results indicated that a predominant number of A. baumannii strains were isolated from pulmonary samples (with a rate of $36.84 \%$ ) and from the intensive care unit (57.89\%). 


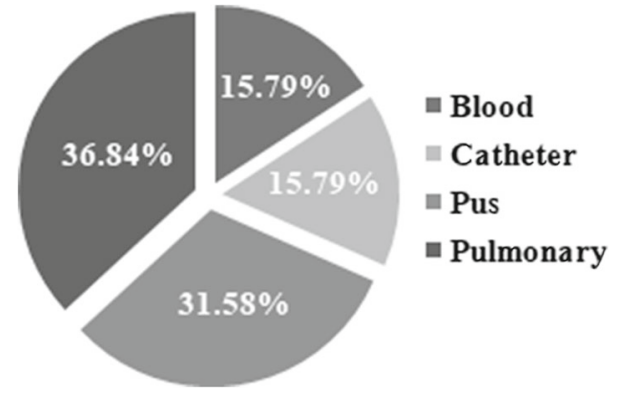

Fig. 1 Distribution of A. baumannii strains according to the nature of samples

This observation was consistent with the results reported by Ben Haj Khalifa and Khedher [17] who stated the airway as the main isolation site for this species. Furthermore, Delbos [18] reported that hospital-acquired pneumonia is still the most common infection caused by A. baumannii. Baranzelli et al. [19] showed that the incidence of lower respiratory tract infections related to Stenotrophomonas maltophilia and A. baumannii is increasing, especially in intensive care units. These two Gram-negative bacilli are mainly identified in nosocomial infections such as ventilator-associated pneumonia, bacteremia and surgical wound infection. Between 2017 and 2019, several authors have shown the impact of A. baumannii in pulmonary infections. Tan et al. [20] reported that bacteremic pneumonia caused by $A$. baumannii has a higher mortality compared to other microorganisms. Wong et al. [21] reported that in Asian and certain Latin American countries, A. baumannii is one of the three most common causes of bacteremia and nosocomial pneumonia and in one wellpublicized case, a health care worker developed fulminant pneumonia after inhalation of A. baumannii aerosolized during endotracheal suctioning of a ventilated patient. Huang et al. [22] reported that $A$. baumannii has been considered to be a prevailing pathogen causing ventilator-associated pneumonia (VAP), leading to high morbidity and mortality among patients in several intensive care unit (ICUs). In a retrospective study, these authors assessed clinical characteristics and antimicrobial resistance of A. baumannii in VAP on the basis of the patients between the year of 2015 and 2017. They found that the incidence of A. Baumannii-VAP was approximately $35.9 \%$ in all VAP patients. Finally, from the molecular point of view, Martínez-Guitián et al. [23] showed that the hisF gene from A. baumannii ATCC 17978, found as over-expressed during a murine pneumonia infection, is involved in virulence, which places it as a new potential target for antimicrobial therapies.

Our results showed also that the presence of A. baumannii was involved in $31.58 \%$ of pus samples. This type of sample came from hospitalized patients who had undergone surgery. A similar rate (32.5\%) was obtained by Falagas et al. [24]. However, a study carried out by Sileem et al. [25] revealed a
Table 1 Distribution of A. baumannii strains according to the provenance of samples

\begin{tabular}{llll}
\hline $\begin{array}{l}\text { Provenance } \\
\text { of } \\
\text { samples }\end{array}$ & $\begin{array}{l}\text { Number of A. baumannii } \\
\text { strains }\end{array}$ & Rate of A. baumannii (\%) \\
\hline Hospital & Medical reanimation & 11 & 78.94 \\
& Pediatric department & 2 & \\
& Nephrology department & 2 & \\
Extern & & 4 & 21.05 \\
Total & & 19 & 100 \\
\hline
\end{tabular}

rate of $3.8 \%$ which was lower than that found in our study. A. baumannii strains were also isolated from blood and catheter samples, each with a rate of $15.79 \%$. Research done by Uwingabiye et al. [26] indicated the presence of A. baumannii in $14.51 \%$ of blood samples, but according to Cisneros and Rodriguez Bano [27], A. baumannii was involved in only $1.3 \%$ of septicemia (rate recorded for a period of 7 years: from 1995 to 2002). Catheters colonized by A. baumannii were reported with a low rate of $3.85 \%$ by Uwingabiye et al. [26].

\subsection{Distribution of A. baumannii Strains According to Origin of Samples}

A. baumannii strains were isolated from different samples, especially those received from hospitalized patients with a frequency of $72.7 \%$ (15). Four strains $(21.05 \%)$ came from outpatients who had been recently hospitalized, confirming the nosocomial nature of $A$. baumannii infections as reported by Bennett et al. [28] and Almasaudi [29].

Among the strains isolated from hospitalized patients, the medical reanimation department occupied first position with $57.89 \%$ of isolated strains, followed by the nephrology and the pediatric departments, each with a percentage of $10.52 \%$ (Table 1). A study carried out in Casablanca from 2001 to 2003, showed that $50 \%$ of A. baumannii strains came from the intensive care units [30].

\subsection{Antibiotic Resistance Profile of Acinetobacter baumannii Strains}

The antibiotic resistance profile of A. baumannii strains was determined using the disk diffusion technique as shown in Fig. 2.

Some strains were total resistant to all the 11 antibiotics tested while others were highly resistant (Table 2). Our results showed that all A. baumannii strains were resistant to all $\beta$-lactams, except for $19.09 \%$ strains that were sensitive to imipenem. However, lower resistance rates were recorded in Tunisia in 2009, where resistance to ticarcillin, piperacillin 
Fig. 2 Antibiogram result of $A$. baumannii strain S439. AK amikacin, $T M$ tobramycin, $C I P$ ciprofloxacin, $G N$ gentamicin, $D O$ doxycycline, $S X T$ cotrimoxazole, $I M P$ imipenem, $T I M$ ticarcillin + clavulanic acid, $C A Z$ ceftazidime, $T I C$ ticarcillin, $P I P$ piperacillin, $C L$ colistin

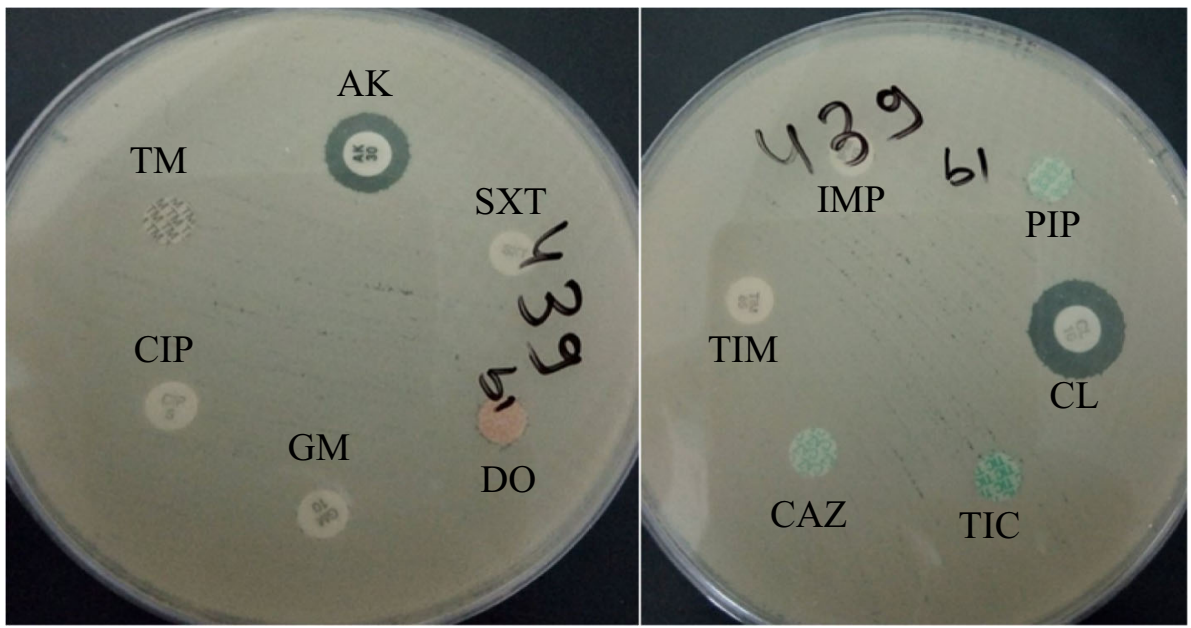

Table 2 Antibiotic resistance profile of A. baumannii

\begin{tabular}{|c|c|c|c|c|c|c|c|c|c|c|c|c|}
\hline \multirow{2}{*}{$\begin{array}{l}\text { Code of A. baumannii } \\
\text { strains }\end{array}$} & \multicolumn{5}{|c|}{$\beta$-lactams } & \multicolumn{3}{|c|}{ Aminosides } & \multirow{2}{*}{$\begin{array}{l}\text { Cyclines } \\
\text { DO }\end{array}$} & \multirow{2}{*}{$\begin{array}{l}\text { Sulfamides } \\
\text { SXT }\end{array}$} & \multirow{2}{*}{$\begin{array}{l}\text { Quinolones } \\
\text { CIP }\end{array}$} & \multirow{2}{*}{$\begin{array}{l}\text { Polypeptides } \\
\text { CL }\end{array}$} \\
\hline & TIC & TCC & PIP & CAZ & IMP & $\mathrm{AK}$ & GN & $\mathrm{TM}$ & & & & \\
\hline $\begin{array}{l}\text { S1804, S439, S292 S483, } \\
\text { S608, S460, S457, S54, } \\
\text { S341, S214, S361 and } \\
\text { S983 }\end{array}$ & $\mathrm{R}$ & $\mathrm{R}$ & $\mathrm{R}$ & $\mathrm{R}$ & $\mathrm{R}$ & $\mathrm{R}$ & $\mathrm{R}$ & $\mathrm{R}$ & $\mathrm{R}$ & $\mathrm{R}$ & $\mathrm{R}$ & S \\
\hline S1816 & $\mathrm{R}$ & $\mathrm{R}$ & $\mathrm{R}$ & $\mathrm{R}$ & $\mathrm{R}$ & $\mathrm{R}$ & $\mathrm{R}$ & $\mathrm{R}$ & $\mathrm{R}$ & $\mathrm{S}$ & $\mathrm{R}$ & S \\
\hline S344 & $\mathrm{R}$ & $\mathrm{R}$ & $\mathrm{R}$ & $\mathrm{R}$ & $\mathrm{S}$ & $\mathrm{S}$ & $S$ & $\mathrm{R}$ & $\mathrm{R}$ & $\mathrm{S}$ & $\mathrm{S}$ & $S$ \\
\hline S136 & $\mathrm{R}$ & $\mathrm{R}$ & $\mathrm{R}$ & $\mathrm{R}$ & $\mathrm{S}$ & $S$ & $\mathrm{R}$ & $\mathrm{R}$ & $S$ & $\mathrm{~S}$ & $\mathrm{R}$ & $S$ \\
\hline S568 & $\mathrm{R}$ & $\mathrm{R}$ & $\mathrm{R}$ & $\mathrm{R}$ & $\mathrm{S}$ & $S$ & $S$ & $\mathrm{R}$ & $\mathrm{S}$ & $\mathrm{S}$ & $\mathrm{S}$ & $S$ \\
\hline S581 & $\mathrm{R}$ & $\mathrm{R}$ & $\mathrm{R}$ & $\mathrm{R}$ & $\mathrm{S}$ & $\mathrm{S}$ & $S$ & $\mathrm{R}$ & $\mathrm{R}$ & $\mathrm{S}$ & $\mathrm{S}$ & $S$ \\
\hline S01 and S1000 & $\mathrm{R}$ & $\mathrm{R}$ & $\mathrm{R}$ & $\mathrm{R}$ & $\mathrm{R}$ & $\mathrm{R}$ & $\mathrm{R}$ & $\mathrm{R}$ & $\mathrm{R}$ & $\mathrm{S}$ & $\mathrm{R}$ & $\mathrm{S}$ \\
\hline
\end{tabular}

B-lactams: (TIC ticarcillin, TCC ticarcillin + clavulanic acid, PIP piperacillin, CAZ ceftazidime, IMP imipenem), aminosides: (AK amikacin, $G N$ gentamicin, $T M$ tobramycin), $D O$ doxycycline, $S X T$ cotrimoxazole, $C I P$ ciprofloxacin, $C L$ colistin, $R$ resistant, $S$ sensible

and ceftazidime was 51.7, 58.4 and 52.2\%, respectively [17]. Grati et al. [31] reported the imipenem resistance rate as $87.5 \%$ which is close to our results $(81.81 \%)$. In our study, the resistance of A. baumannii to aminoglycosides was recorded as $100 \%$ for tobramycin and exceeded $80 \%$ for gentamicin. These resistance rates were high compared to those found in Europe, which were $47.1 \%$ for gentamicin [32], and $41 \%$ for tobramycin [33]. Concerning the resistance to ciprofloxacin, a rate of $86.36 \%$ was noted in our study, which was close to that recently stated in Morocco (91\%) [34]. These results allowed us to consider our strains as MDR (multidrug resistant) [35]. Lastly, all strains were sensitive to colistin with MICs not exceeding $1 \mu \mathrm{g} / \mathrm{ml}$. Our results were similar to those obtained by Kandeel [36], who stated the MIC of colistin as less than $2 \mu \mathrm{g} / \mathrm{ml}$.

\subsection{Phenotypes of Antibiotic Resistance Mechanisms Characterized in A. baumannii}

The antibiogram complimentary tests phenotypically revealed three enzymatic mechanisms of resistance in the tested $A$. baumannii strains: extended-spectrum $\beta$-lactamases (ESBL), cephalosporinase and metallo- $\beta$-carbapenemases as shown in Table 3.

Only three strains (15.79\%), out of 19 tested, were ESBL producers. According to the national surveillance for resistant bacteria done in Algeria in 2009, the production of ESBL was $22.02 \%$ in 16 tested laboratories which show that the number of $A$. baumannii ESBL producers remains low [37]. Furthermore, screening of these strains for cephalosporinases revealed that 10 strains $(52.63 \%)$ of A. baumannii were cephalosporinase positive. Yongrui and Xiangqun [38] revealed a higher rate (72\%). The phenotypic tests EDTA and Hodge highlighted the production of metallo$\beta$-carbapenemases by 15 strains $(78.95 \%)$, similar to a rate that was found by Kaur et al. [39] (80.3\%). On the other hand, 
Table 3 Resistance phenotypes of A. baumannii

\begin{tabular}{llll}
\hline $\begin{array}{l}\text { Code of } A . \\
\text { baumannii } \\
\text { strains }\end{array}$ & ESBL & $\begin{array}{l}\text { Metallo- } \beta \text { - } \\
\text { carbapenemase }\end{array}$ & Cephalosporinase \\
\hline S54 and S893 & + & + & + \\
S01, S214, & - & + & - \\
S292, S341, & & & \\
S344, S439, & & & \\
S457, S460 & & & + \\
and S1000 & & & \\
S136, S361, & - & + & + \\
S483 and & & & + \\
S581 & & & + \\
S568, S608 & - & - & + \\
and S1804 & & & \\
S1816 & + & - & \\
\hline
\end{tabular}

+ Present, - absent

lower rates (between 22 and 49\%) of A. baumannii metallo$\beta$-lactamase positive strains were reported by many authors [40-42].

\subsection{Evaluation of the Antibacterial Potential of Actinobacterial Isolates}

Fifty-nine actinobacterial isolates cultured on ISP2 medium were tested against 19 A. baumannii strains by agar plug diffusion method to evaluate their antibacterial potential. The antibacterial activity screening revealed that 19 (32.20\%) isolates were active against at least one A. baumannii strain. In contrast, $40(67.80 \%)$ isolates showed no activity. An antibacterial activity was obtained by all 13 Saccharothrix (or Saccharothrix-like) isolates, followed by five Actinomadura isolates and one isolate of Streptomyces. The results of these active isolates are recorded in Table 4.

The antagonistic activity against $A$. baumannii obtained in the 19 active isolates was generally strong for the majority of Saccharothrix (or Saccharothrix-like) isolates and the single Streptomyces isolate (WAB9). It was average for the five Actinomadura isolates. The diameters of inhibitions obtained by these active isolates varied between 10 and $30 \mathrm{~mm}$. We noticed a variation in diameters of inhibition between isolates of the same genus; those of Saccharothrix (or Saccharothrix-like) ranged from 11 to $30 \mathrm{~mm}$, whereas those of Actinomadura ranged from 10 to $22 \mathrm{~mm}$.

The Saccharothrix isolates were highly active with a maximum inhibition diameter of $30 \mathrm{~mm}$ against some $A$. baumannii strains. In contrast, in the scientific literature, most antibiotics secreted by Saccharothrix isolates mainly target Gram-positive bacteria and fungi, and are rarely directed against Gram-negative bacteria [43-47].
Secondly, the Streptomyces sp. WAB9 followed with a maximum inhibition zone of $25 \mathrm{~mm}$. Our results were similar to those of Yekkour et al. [48], who stated that this strain of Streptomyces sp. has a broad spectrum that extends over several filamentous fungi, and a large number of pathogenic bacteria (Gram-positive and Gram-negative), which are multi-resistant to several antibiotics. These authors found that the high activity of Streptomyces WAB9 (close to the species Streptomyces ambofaciens) is due to the production of a new antibiotic (named W9), which is a hydroxamic acid-containing molecule. Hydroxamic acidcontaining molecules are known to exhibit low toxicities in general, which is very interesting.

Finally, Actinomadura was active with a maximum inhibition zone of $22 \mathrm{~mm}$. Actinomadura isolates are known for the production of several antibacterial molecules, namely maduramicin, actinotiocin and carminomycin secreted, respectively, by A. rubra, A. pusilla and A. carminata [49].

The action spectrum of the antibacterial molecules secreted by the active isolates was quite broad for the majority of isolates with a maximal spectrum obtained by Streptomyces $\mathrm{sp}$. WAB9.

\section{Conclusion}

During the course of this study, nineteen A. baumannii strains were obtained from various clinical samples at Béni Messous University Hospital Center (Algiers, Algeria). The majority of the strains were isolated from pulmonary samples received from patients hospitalized in the intensive care unit. The evaluation of antibiotic resistance of these strains against antibiotics recommended for treatment of A. baumannii infections revealed the ineffectiveness of the majority of the molecules. We noted a total absence of $\beta$-lactam antibacterial activity against all the strains. This was attributed to the production of different enzymes that degraded the antibiotic molecules namely metallo- $\beta$-carbapenemases and cephalosporinases. Nonetheless, all the A. baumannii strains were sensitive to colistin which was active at very low MICs. The results from the antagonistic potential evaluation carried out on a collection of Actinobacteria showed the ability of certain isolates belonging to the genera Saccharothrix, Streptomyces and Actinomadura to secrete bioactive molecules against $A$. baumannii. Thus, faced with this $A$. baumannii antibacterial resistance difficulty and a limited number of active molecules against it, Actinobacteria could be an alternative source of bioactive molecules that could meet this increased necessity. 


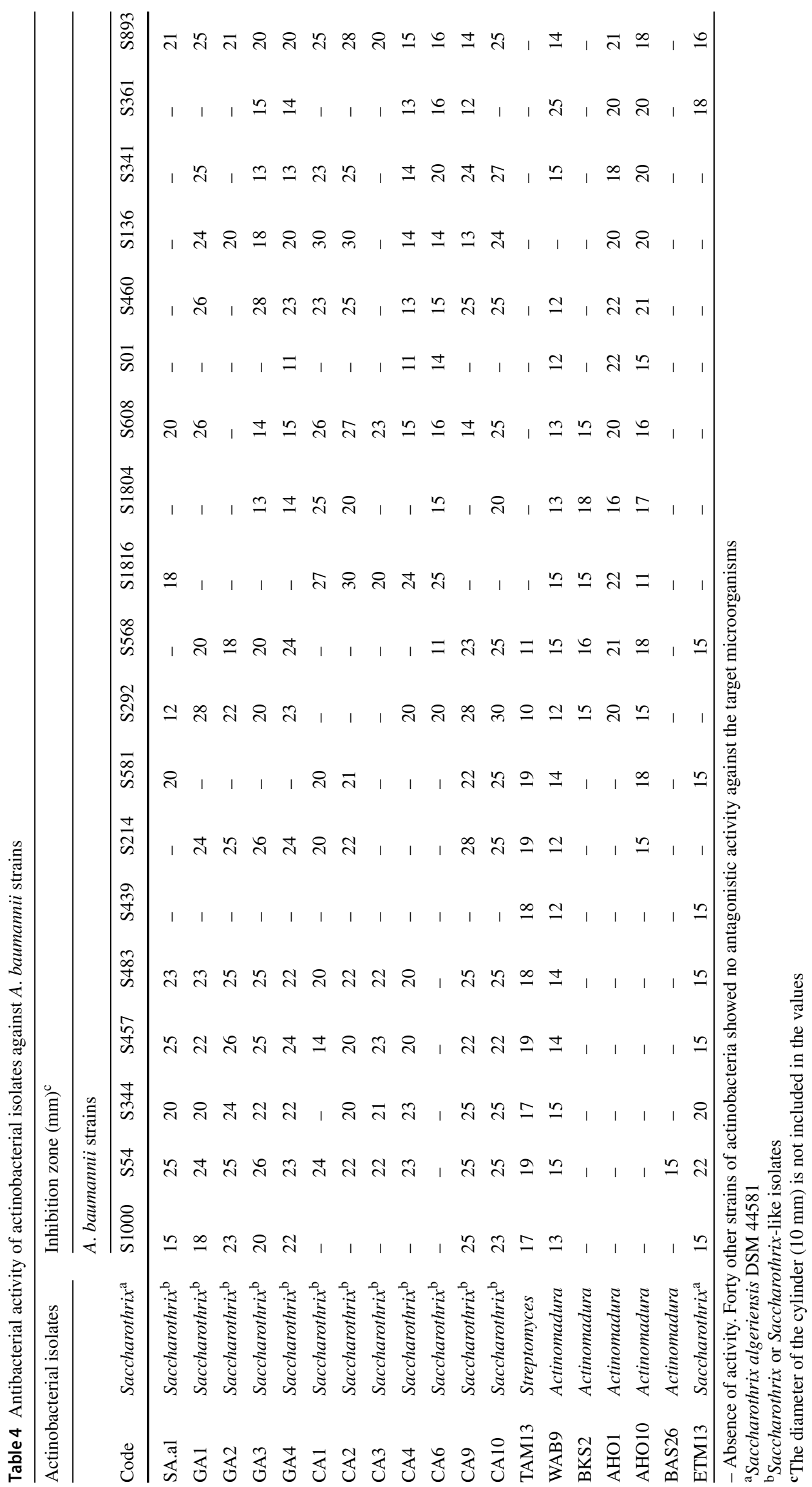

I Springer 


\section{References}

1. Decré, D.S.: Acinetobacter baumannii et résistance aux antibiotiques un modèle d'adaptation. RFL 441, 43-52 (2012)

2. McConnell, M.J.; Actis, L.; Pachon, J.: Acinetobacter baumannii: human infections, factors contributing to pathogenesis and animal models. FEMS Microbiol. Rev. 37, 130-155 (2012)

3. Peleg, A.Y.; Seifert, H.; Paterson, D.L.: Acinetobacter baumannii: emergence of a successful pathogen. Clin. Microbiol. Rev. 21, 538-582 (2008)

4. Obeidat, N.; Jawdat, F.; Al-Bakri, A.G.; Shehabi, A.A.: Major biologic characteristics of Acinetobacter baumannii isolates from hospital environmental and patients' respiratory tract sources. Am. J. Infect. Control 42, 401-404 (2014)

5. Gonzalez-Villoria, A.M.; Valverde-Garduno, V.: Antibioticresistant Acinetobacter baumannii increasing success remains a challenge as a nosocomial pathogen. J. Pathog. 45, 1-10 (2006)

6. George, M.; Anjumol, A.; George, G.; Mohamed Hatha, A.A.: Distribution and bioactive potential of soil actinomycetes from different ecological habitats. Afr. J. Microbiol. Res. 6, 2265-2271 (2012)

7. Solecka, J.; Zajko, J.; Postek, M.; Rajnisz, A.: Biologically active secondary metabolites from actinomycetes. Open Life Sci. 7, 373-390 (2012)

8. Jiang, Y.; Li, Q.; Chen, X.; Jiang, C.: Isolation and cultivation methods of Actinobacteria. In: Dhanasekaran, D., Jiang, Y. (eds.) Actinobacteria-Basics and Biotechnological Applications. IntechOpen, London (2016)

9. Prince, C.P.: Practical Manual of Medical Microbiology, pp. 112-114. Jaypee Brothers Medical Publishers, New Delhi (2009)

10. Denis, F.; Poly, M.C.; Martin, C.; Bingen, E.; Quentin, R.: Bactériologie Medicale. Techniques Usuelles, p. 75. Elsevier, Paris (2011)

11. Hidri, N.: Identification d'Acinetobacter spp. au laboratoire. FRL 441, 37-41 (2012)

12. Joffin, J.N; Leyral, G.: Microbiologie Technique. Tome 1, Dictionnaire des techniques. Ed. Scérén, p. 150 (2014)

13. Rahal, K.; Tali-Maamar, H.; Missoum, M.F.K.; Aboun, A.: Standardisation des Tests de sensibilité aux antibiotiques à l'échelle nationale (Médecine humaine et vétérinaire), vol. $7^{\text {ème }}$,pp. 2-179 (2014)

14. Patel, J.J.; Brown, E.M.: Interactions of Azotobacter with rhizosphere and root-surface microflora. Plant Soil 31, 273-281 (1969)

15. Daef, E.A.; Mohamad, I.S.; Ahmad, A.S.; El-Gendy, S.G.; Ahmed, E.H.; Sayed, I.M.: Relationship between clinical and environmental isolates of Acinetobacter baumannii in Assiut University Hospitals. J. Am. Sci. 9, 67-73 (2013)

16. Henein, A.E.: The Potential of Bacteriophage Therapy in Acinetobacter spp. Infections. Ph.D. Thesis, University of Brighton, p. 523 (2009)

17. Ben Haj Khalifa, A.; Khedher, M.: Profil de sensibilité aux antibiotiques des souches d'Acinetobacter baumannii isolées dans la région de Mahdia. Med. Mal. Infect. 40, 126-128 (2010)

18. Delbos, V.: Manifestations cliniques et traitement des infections à Acinetobacter baumannii. RFL 441, 59-65 (2012)

19. Baranzelli, A.; Wallyn, F.; Nseir, S.: Infections bronchopulmonaires à Stenotrophomonas maltophilia et à Acinetobacter baumannii. Rev. Pneumol. Clin. 69, 250-259 (2013)

20. Tan, Y.; Zhou, K.; Tang, X.; Kudinha, T.; Wang, L.; Guo, Z.; Akova, M.T.; Zhuo, C.: Bacteremic and non-bacteremic pneumonia caused by Acinetobacter baumannii in ICUs of South China: a clinical and microbiological study. Sci. Rep. 7, 15279 (2017). https://doi.org/ 10.1038/s41598-017-13148-y

21. Wong, D.; Nielsen, T.B.; Bonomo, R.A.; Pantapalangkoor, P.; Luna, B.; Spellberg, B.: Clinical and pathophysiological overview of Acinetobacter infections: a century of challenges. Clin. Microbiol. Rev. 30, 409-447 (2017)

22. Huang, Y.; Zhou, Q.; Wang, W.; Huang, Q.; Liao, J.; Li, J.; Long, L.; Ju, T.; Zhang, Q.; Wang, H.; Xu, H.; Tu, M.: Acinetobacterbaumannii ventilator-associated pneumonia: clinical efficacy of combined antimicrobial therapy and in vitro drug sensitivity test results. Front. Pharmacol. (2017). https://doi.org/10.3389/fphar.2019.00092

23. Martínez-Guitián, M.; Vázquez-Ucha, J.C.; Álvarez-Fraga, L.; Conde-Pérez, K.; Vallejo, J.A.; Bou, G.; Poza, M.; Beceiro A.: Implication of HisF from Acinetobacter baumannii in persistence during a pneumonia infection (2019). https://doi.org/10. $1101 / 534966$ (in press)

24. Falagas, E.M.; Vardakas, Z.K.; Kapaskelis, A.; Triarides, A.N.; Roussos, N.: Tetracycline for multidrug-résistant Acinetobacter Baumannii infections. Int. J. Antimicrob. Agents 14, 252-258 (2015)

25. Sileem, A.E.; Said, A.M.; Meleha, M.S.: Acinetobacter baumannii in ICU patients: A prospective study highlighting their incidence, antibiotic sensitivity pattern and impact on ICU stay and mortality. Egypt J. Chest Dis. Tuberc. 66, 693-698 (2017)

26. Uwingabiye, J.; Frikh, M.; Lemnouer, A.; Bssaibis, F.; Belefquih, B.; Maleb, A.; Dahraoui, S.; Belyamani, L.; Bait, A.; Haimeur, C.; Louzi, L.; Ibrahimi, A.: Acinetobacter infections prevalence and frequency of the antibiotics resistance: comparative study of intensive care units versus other hospital units. PAMJ 23, 191-197 (2016)

27. Cisneros, M.J.; Rodríguez-Baño, J.: Nosocomial bacteremia due to Acinetobacter baumannii: epidemiology, clinical features and treatments. Clin. Microbiol. Infect. 8, 687-693 (2002)

28. Bennett, J.E; Dolin, R.; Blaser, M.J.: Mandell, Douglas and Bennett's infectious diseases essentials, vol. 1, p. 3904 (2016). ISBN:978-1-4557-4801-3

29. Almasaudi, S.: Acinetobacter spp. as nosocomial pathogens: epidemiology and resistance features. Saudi J. Biol. Sci. 25, 586-596 (2018)

30. Elouennass, M.; Bajou, T.; Lemnouer, A.H.; Foissaud, V.; Hervé, V.; Baaj, A.J.: Acinetobacter baumannii: étude de la sensibilité des souches isolées à l'hôpital militaire d'instruction Mohammed V, Rabat, Maroc. Méd. Mal. Infect. 33, 361-364 (2003)

31. Grati,L.; Louzi, M.; Mansalli, L.; Ben Nasr, K.;Zili, N.; Ben Salem, F.; Gahbiche, M.: Les pneumopathies nosocomiales en réanimation chirurgicale. Une année de surveillance portant sur 305 patients. J. Magh. A. Réa. Méd. Urg. 7, 26-29 (2005)

32. Unal, S.; Garcia-Rodriguez, J.A.: Activity of meropenem and comparators against Pseudomonas aeruginosa and Acinetobacter spp. isolated in the MYSTIC program, 2002-2004. Diagn. Microbiol. Infect. Dis. 53, 265-271 (2005)

33. Turner, P.J.; Greenhalgh, J.M.: MYSTIC Study Group (Europe): the activity of meropenem and comparators against Acinetobater strains isolated from European Hospitals, 1997-2000. Clin. Microbiol. Infect. 9, 563-567 (2000)

34. Khaldi, H.: Epidémiologie de l'infection à Acinetobacter baumannii au CHU de Marrakech, p. 131. Doctorat en médecine, Marrakech (2016)

35. Siegel, J.D.; Rhinehart, E.; Jackson, M.; Chiarello, L.: Guideline for isolation precautions: preventing transmission of infectious agents in healthcare settings. J. Infect. Dis. 24, 218-230 (2006)

36. Kandeel, A.: Detection of colistin susceptibility in multi-drug resistant Pseudomonas aeruginosa and Acinetobacter baumannii by four different methods. Egypt J. Med. Microbiol. 25, 17-23 (2016)

37. Benslimani, A.: MRSA, entérobactéries BLSE, Acinetobacter sp. et Pseudomonas aeruginosa résistants à l'imipénème, à la céftazidime et à la ciprofloxacine. In: Surveillance de la résistance des bactéries aux antibiotiques. $11^{\text {ème }}$ rapport d'évaluation, p. 198 (2009) 
38. Yongrui, L.; Xiangqun, L.: Detection of AmpC beta-lactamase in Acinetobacter baumannii in the Xuzhou region and analysis of drug resistance. Exp. Ther. Med. 10, 933-936 (2015)

39. Kaur, A.; Gupta, V.; Chhina, D.: Prevalence of metallo- $\beta$-lactamase producing (MBL) Acinetobacter species in a tertiary care hospital. Iran J. Microbiol. 6, 22-25 (2014)

40. Peymani, A.; Nahaei, M.R.; Farajnia, S.; Hasani, A.; Mirsalehian, A.; Sohrabi, N.; Abbasi, L.: High prevalence of metallobeta-lactamase-producing Acinetobacter baumannii in a teaching hospital in Tabriz, Iran. Jpn. J. Infect. Dis. 64, 69-71 (2011)

41. Gildas, A.; Zohoun, C.; Moket, D.; El Hamzaoui, S.: Résistance à l'imipénème par production de métallo- $\beta$-lactamases par Acinetobacter baumannii et Pseudomonas aeruginosa à l'Hôpital militaire d'instruction Mohammed V de Rabat. Ann. Biol. Clin. 71, 27-30 (2013)

42. Mathlouthi, N.; El Salabi, A.A.; Ben Jomaa-Jemili, M.; Bakour, S.; Al-Bayssari, C.; Zorgani, A.A.; Kraiema, A.; Elahmur, O.; Okdah, L.; Rolain, J.M.; Chouchani, C.: Early detection of metallo- $\beta$ lactamase NDM-1-and OXA-23 carbapenemase producing Acinetobacter baumannii in Libyan hospitals. Int. J. Antimicrob. Agents 48, 46-50 (2016)

43. Takeuchi, M.; Takahashi, S.; Enokita, R.; Sakaida, Y.; Haruyama, H.; Nakamura, T.; Katayama, T.; Inukai, M.: Galacardins A and B, new glycopeptide antibiotics. J. Antibiot. 45, 297-305 (1992)
44. Kinoshita, N.; Igarashi, M.; Ikeno, S.; Hori, M.; Hamada, M.: Saccharothrix tangerinus sp. nov., the producer of the new antibiotic formamicin: taxonomic studies. Actinomycetologica 13, 20-31 (1999)

45. Wang, Z.X.; Li, S.M.; Heide, L.: Identification of the coumermycin A1 biosynthetic gene cluster of Streptomyces rishiriensis DSM 40489. Antimicrob. Agents Chemother. 44, 3040-3048 (2000)

46. Lamari, L.; Zitouni, A.; Dob, T.; Sabaou, N.; Lebrihi, A.; Germain, P.; Seguin, E.; Tillequin, F.: New dithiolopyrrolone antibiotics from Saccharothrix sp. SA 233. II. Physicochemical properties and structure elucidation. J. Antibiot. 55, 702-706 (2002)

47. Zitouni, A.; Boudjella, H.; Lamari, L.; Badji, B.; Mathieu, F.; Lebrihi, A.; Sabaou, N.: Nocardiopsis and Saccharothrix genera in Saharan soils in Algeria: isolation, biological activities and partial characterization of antibiotics. Res. Microbiol. 156, 984-993 (2005)

48. Yekkour, A.; Meklat, A.; Bijani, C.; Toumatia, O.; Errakhi, R.; Lebrihi, A.; Mathieu, F.; Zitouni, A.; Sabaou, N.: A novel hydroxamic acid-containing antibiotic produced by a Saharan soil-living Streptomyces strain. Lett. Appl. Microbiol. 60, 589-596 (2015)

49. Solanki, R.; Khanna, M.; Lal, R.: Bioactive compounds from marine actinomycetes. Indian J. Microbiol. 48, 410-431 (2008) 